\title{
ON COMPLETE LATTICES HAVING THE HAUSDORFF INTERVAL TOPOLOGY
}

\author{
KOICHI ATSUMI
}

Recently Arnold J. Insel [1] established a necessary and sufficient condition for a lattice to be Hausdorff in its complete topology, namely that every net in the lattice has an order convergent subnet. The purpose of the present note is to show that this condition is also necessary and sufficient for a lattice to be compact and Hausdorff in its interval topology. An immediate consequence of this result is that if a complete lattice is Hausdorff in its interval topology, then the lattice is compact and Hausdorff in its order topology and in fact two topologies are the same. This can be shown easily using the results of Insel [1], but we give here the proof without making use of the complete topology defined by Insel.

Let $L$ be a lattice and $\left\{x_{a}: a \in A\right\}$, a net in $L$. If $\vee_{a} \wedge\left\{x_{b}: b \geqq a\right\}$ $=\wedge_{a} \bigvee\left\{x_{b}: b \geqq a\right\}=x$, we say that the net $\left\{x_{a}: a \in A\right\}$ order converges to $x$. A set $F$ of $L$ is called order closed if no net in $F$ order converges to a point outside of $F$. The collection of order closed sets comprises the closed sets for a topology for $L$. This topology is called the order topology of $L$ and is designated by $O(L)$. The collection of sets of the form $\{x: x \leqq t\}$ and $\{x: t \leqq x\}$ for $t \in L$ forms the subbase of the closed sets of a weaker topology called the interval topology and designated by $I(L)$. It is well known that $I(L)$ is compact iff $L$ is a complete lattice.

TheOREM 1. Let $L$ be a complete lattice and $\left\{x_{a}: a \in A\right\}$, a net in $L$. Then $\left\{x_{a}: a \in A\right\}$ converges to a point $x$ with respect to the interval topology, iff $\vee_{c} \wedge\left\{x_{c}: c \in C\right\} \leqq x \leqq \wedge_{c} \vee\left\{x_{c}: c \in C\right\}$, where $C$ denotes an arbitrary cofinal subset of $A$.

Proof. Suppose that $\left\{x_{a}: a \in A\right\}$ converges $x$ in $I(L)$ and that $\vee_{c} \wedge\left\{x_{c}: c \in C\right\} \leq x$, or $x \leq \wedge \vee\left\{x_{c}: c \in C\right\}$. If $\vee_{c} \wedge\left\{x_{c}: c \in C\right\} \leq x$, there exists a cofinal set $C_{0}$ of $A$ such that $\Lambda\left\{x_{c}: c \in C_{0}\right\}$. Then $U=L-\left\{z: \wedge\left\{x_{c}: c \in C_{0}\right\} \leqq z\right\}$ is a $I(L)$-open neighborhood of $x$, and by hypothesis $\left\{x_{a}: a \in A\right\}$ is eventually in $U$. But this contradicts the fact that $\left\{x_{c}: c \in C_{0}\right\}$ is not contained in $U$. Similarly $x \neq \wedge_{c} \vee\left\{x_{c}: c \in C\right\}$ gives a contradiction.

To prove the converse, suppose that $\vee_{c} \wedge\left\{x_{c}: c \in C\right\} \leqq x$ $\leqq \wedge_{c} \vee\left\{x_{c}: c \in C\right\}$, and that $\left\{x_{a}: a \in A\right\}$ does not converge to $x$ with respect to $I(L)$. Let $U$ be a $I(L)$-open neighborhood of $x$. Then

Received by the editors March 6, 1965. 
$\left\{x_{a}: a \in A\right\}$ is frequently in $L-U$. But $L-U$ is an intersection of a family of members of the closed base for $I(L)$. Hence there exist a set $F$ of this closed base such that $x \notin F$ and $\left\{x_{a}: a \in A\right\}$ is frequently in $F$. But $F$ is the form $\cup\left\{J_{i}: i=1,2, \cdots, n\right\}$, where $J_{i}$ is a set of the form $\{z: z \leqq t\}$ or $\{z: t \leqq z\}$ for $t \in L$. Consequently there exists a cofinal set $C_{0} \subset A$, such that $\left\{x_{c}: c \in C_{0}\right\} \subset J_{i}$ holds for some $i$. If $J_{i}=\{z: t \leqq z\}$, then $t \leqq \Lambda\left\{x_{c}: c \in C_{0}\right\}$ and $t \leqq \vee_{c} \wedge\left\{x_{c}: c \in C\right\} \leqq x$, this contradicts the fact that $x \in J_{i}$. On the other hand, if $J_{i}=\{z: z \leqq t\}$, then $\vee\left\{x_{c}: c \in C_{0}\right\} \leqq t$ and $x \leqq \wedge_{c} \vee\left\{x_{c}: c \in C\right\} \leqq t$, hence $x \in J_{i}$, a contradiction.

We have immediately the following two corollaries:

Corollary 1. In a complete lattice $L, a$ net $\left\{x_{a}: a \in A\right\}$ converges with respect to $I(L)$, iff $\vee_{c} \wedge\left\{x_{c}: c \in C\right\} \leqq \wedge_{c} \vee\left\{x_{c}: c \in C\right\}$.

Corollary 2. Let $L$ be a complete lattice. The interval topology $I(L)$ of $L$ is Hausdorff, iff $\vee_{c} \wedge\left\{x_{c}: c \in C\right\} \leqq \wedge_{c} \vee\left\{x_{c}: c \in C\right\}$ implies $\vee_{c} \wedge\left\{x_{c}: c \in C\right\}=\wedge_{c} \vee\left\{x_{c}: c \in C\right\}$.

For any topological space $X$ a net $\left\{x_{a}: a \in A\right\}$ is called a universal net if it is eventually in or eventually outside of any subset of $X$. Every net has a universal subnet. $X$ is compact iff every universal net in $X$ converges, and $X$ is Hausdorff iff no universal net in $X$ converges to more than one point [2].

THEOREM 2. Let $\left\{x_{a}: a \in A\right\}$ be a universal net in L. We have two equalities $\vee_{a} \wedge\left\{x_{b}: b \geqq a\right\}=\vee_{c} \wedge\left\{x_{c}: c \in C\right\}, \wedge_{a} \vee\left\{x_{b}: b \geqq a\right\}$ $=\wedge_{c} \vee\left\{x_{c}: c \in C\right\}$, provided each member exists. Here $C$ denotes an arbitrary cofinal subset of $A$.

Proof. Let $C_{0}$ be any cofinal subset of $A$ and consider $J=\left\{z: \wedge\left\{x_{c}: c \in C_{0}\right\} \leqq z\right\}$. Since $\left\{x_{a}: a \in A\right\}$ is frequently in $J$, and it is a universal net, there exists an $a_{0}$ such that $\Lambda\left\{x_{c}: c \in C_{0}\right\} \leqq x_{b}$ for all $b \geqq a_{0}$. Consequently $\Lambda\left\{x_{c}: c \in C_{0}\right\} \leqq \Lambda\left\{x_{b}: b \geqq a_{0}\right\} \leqq \vee_{a} \wedge\left\{x_{b}\right.$ : $b \geqq a\}$. Since $C_{0}$ was an arbitrary cofinal subset, we have $\vee_{c} \wedge\left\{x_{c}\right.$ : $c \in C\} \leqq \vee_{a} \wedge\left\{x_{b}: b \geqq a\right\}$. On the other hand, since $\{b \geqq a\}$ is a cofinal subset of $A$, we have evidently $\vee_{a} \wedge\left\{x_{b}: b \geqq a\right\} \leqq \vee_{c} \wedge\left\{x_{c}: c \in C\right\}$. Hence we have the first equality.

The second equality can be shown in a similar way.

Theorem 3. For any lattice $L$ the following are equivalent:

(1) $L$ is compact and Hausdorff in its interval topology.

(2) Every universal net in $L$ is order convergent.

(3) Every net in L has an order convergent subnet.

Proof. (1) implies (2). Let $\left\{x_{a}: a \in A\right\}$ be a universal net in $L$. 
Since $L$ is compact with respect to $I(L),\left\{x_{a}: a \in A\right\}$ converges in the interval topology, and $L$ is a complete lattice. Then we have $\vee_{c} \wedge\left\{x_{a}: c \in C\right\} \leqq \wedge_{c} \vee\left\{x_{c}: c \in C\right\}$ by Corollary 1. Since $L$ is Hausdorff in $I(L)$, we have $\vee_{c} \wedge\left\{x_{c}: c \in C\right\}=\wedge_{c} \vee\left\{x_{c}: c \in C\right\}$ by Corollary 2 , and applying Theorem 2 we have $\vee_{a} \wedge\left\{x_{b}: b \geqq a\right\}$ $=\bigwedge_{a} \bigvee\left\{x_{b}: b \geqq a\right\}$. This shows that $\left\{x_{a}: a \in A\right\}$ is order convergent.

(2) implies (3). This follows directly from the fact that every net has a universal subnet.

(3) implies (1). Let $\left\{x_{a}: a \in A\right\}$ be a net in $L$. Since every net has an order convergent subnet and order convergence implies convergence with respect to $I(L),\left\{x_{a}: a \in A\right\}$ has a subnet which converges with respect to interval topology. Hence $L$ is compact in $I(L)$ and $L$ is a complete lattice.

Let $\left\{x_{d}: d \in D\right\}$ be a universal net which converges to $x$ with respect to $I(L) .\left\{x_{d}: d \in D\right\}$ has an order convergent subnet $\left\{y_{a}: a \in A\right\}$ by hypothesis and $\left\{y_{a}: a \in A\right\}$ converges to $x$ in $I(L)$. Hence $\vee_{c} \wedge\left\{y_{c}: c \in C\right\} \leqq x \leqq \wedge_{c} \vee\left\{y_{c}: c \in C\right\}$, where $C$ denotes any cofinal subset of $A$. Since $\left\{y_{a}: a \in A\right\}$ is universal and order convergent we have $\vee_{c} \wedge\left\{y_{c}: c \in C\right\}=\vee_{a} \wedge\left\{y_{b}: b \geqq a\right\}=\wedge_{a} \vee\left\{y_{b}: b \geqq a\right\}$ $=\wedge c \vee\left\{y_{c}: c \in C\right\}=t$. Hence $x=t$ and $\left\{x_{d}: d \in D\right\}$ converges to one and only one element of $L$ with respect to $I(L)$. Therefore $L$ is Hausdorff in $I(L)$.

COROLlaRy 3. If a complete lattice $L$ is Hausdorff in the interval topology then:

(1) $L$ is compact Hausdorff in the order topology.

(2) The order topology and the interval topology for L coincide.

Proof. (1) follows trivially from Theorem 3. Since $I(L) \subset O(L)$, the identity map of $L$ with its order topology onto $L$ with its interval topology is continuous. Since $L$ is compact in $O(L)$ and Hausdorff in $I(L)$, this identity map is a homeomorphism.

\section{REFERENCES}

1. Arnold J. Insel, $A$ relationship between the complete topology and the order topology of a lattice, Proc. Amer. Math. Soc. 15 (1964), 847-850.

2. John L. Kelley, General topology, Van Nostrand, Princeton, N. J., 1955.

Gunma University, MaEbashi, JaPaN 